\title{
The effect of several cocoa diets on the systemic and intestinal humoral immunity in adult rats
}

\author{
T. Perez-Berezo, S. Ramos-Romero, C. Castellote, A. Franch, F. J. Perez-Cano and M. Castell \\ Department of Physiology, Faculty of Pharmacy, University of Barcelona, Spain and Institut de Recerca en Nutrició \\ i Seguretat Alimentària, University of Barcelona, Spain
}

During the last decade increasing interest has been focused on cocoa mainly due to its high flavonoid content ${ }^{(1)}$. Flavonoids are polyphenolic compounds with recognized antioxidant activity as well as immunomodulatory action ${ }^{(2)}$. Previous studies have shown that cocoaenriched diets affect both the intestinal and systemic immune function in young rats ${ }^{(3)}$.

The aim of the present work was to assess the effect of three cocoa diets on the systemic and intestinal immunoglobulin (Ig) secretion patterns of adult rats. To achieve this, female Wistar rats were randomized into 4 dietary groups: one group was fed standard chow, while the other 3 groups received 2, 5 and 10\% cocoa in pelleted chow for 3 weeks. Faecal and sera samples were collected at days 0,8 and 21 after the beginning of the diet for Ig quantification. At the end of the study, a wash of small intestine was obtained. Sera IgA, IgM, IgG, IgG2a, IgG2b, IgG2c and IgG1 concentrations were quantified by ELISA. Faecal and gut wash IgA concentrations were also determined by ELISA.

Faecal IgA levels increased in the reference group during the study. However, the cocoa-fed rats showed no increased ( $2 \%$ diet) or reduced (5\% and $10 \%$ diet) faecal IgA concentrations. Similarly, gut wash IgA followed the same pattern at the end of the study. Cocoa-fed rats showed no significant changes in sera IgA and IgG concentrations, but IgM levels were found to be decreased after 3 weeks of the $10 \%$ cocoa diet $(P<0.05)$. The study of IgG subclasses showed that IgG1 and IgG2a (Th2-related subclasses) levels decreased after cocoa diets, whereas IgG2b and IgG2c (Th1-related subclasses) did not change, or increased, respectively. In conclusion, cocoa diets decreased both intestinal humoral immunity and Th2 sera-related IgG subclasses.
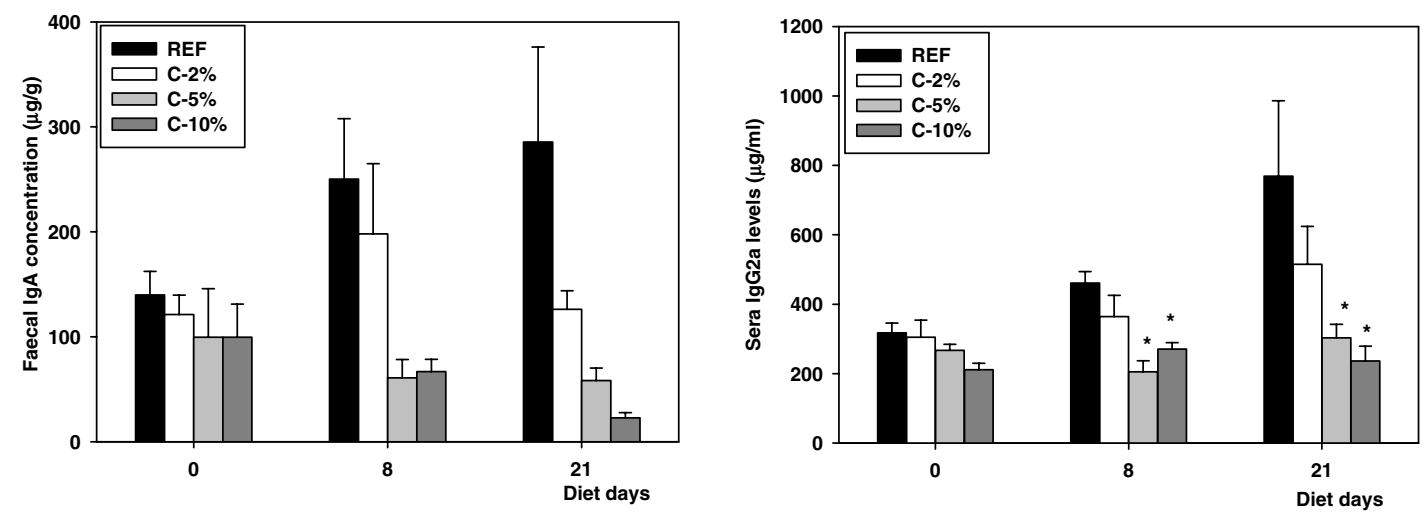

Figure. Effect of cocoa diets on fecal IgA and sera IgG2a concentrations. Each bar represents the means \pm SEM $(n=6-7)$. $* P<0.05$ cocoa groups $v$. standard diet group (U-Mann-Whitney test).

The present study was supported by the Ministerio de Ciencia y Tecnología, Spain (AGL2005-02823 and AGL2008-002790).

1. Visioli F, Bernaert H, Corti R et al. (2009) Crit Rev Food Sci Nutr 49, 299-312.

2. Ramiro-Puig E \& Castell M (2009) Br J Nutr 101, 931-940.

3. Ramiro-Puig E, Pérez-Cano FJ, Ramos-Romero S et al. (2008) J Nutr Biochem 19, 555-565. 